\title{
The treatment with low energy laser in medicine
}

\author{
C. ANTIPA and C. MOLDOVAN* \\ University of Medicine, Coltea Clinical Hospital, No 1 l Bratianu Ave, Bucharest, Romania \\ * April Conmed Ltd., 12 Max Wexler, Bucharest, Romania
}

\section{ABSTRACT}

The paper presents this new therapeutical method, its application fields, hypothesis regarding the acting mechanism at the infracellular, cellular and systemic level, the practical methodology, and some personal results. We finish the paper with general proposals for standardization of the scientific works from this field, which should be necessary due to a large variety of approaching to this matter.

As everybody knows, the surgeons were the first to use lasers in medicine. This kind of lasers, which has a high power (Watts) acts by their thermal effect, vaporizing and coagulating tissues.

In the last years a great number of scientists show themselves interested in using lasers with low power (mWatts) without thermal effect (at least over cellular level). This attraction is justified by the impressive number of papers published all over the world, and especially by the very good results reported in therapeutics.

\section{Definition.}

Dr. C. Galletti defined low power lasers as a source of very low energy, coherent light, without thermal effects upon living tissues. The monochromatic character of the light proved to have a similar importance with coherency. On the other hand, we can find thermal effect at infracellular or molecular levels.

Because we think that is necessary to fix a limited range, even relative, for power used in this kind of lasers, and because we didn't find important studies in this field using lasers with the power over $70 \mathrm{~mW}$, we choosed this limited range betwen 70 and $100 \mathrm{~mW}$.

That's why we proposed this change of the definition: source of very low energy, coherent and monochromatic light, with a power (output power) under $100 \mathrm{~mW}$, without thermal effects at the cellular, organ or organism level.

\section{Terminology.}

It has been used, in the basic or clinical studies, a various number of terms, trying to name this type of laser : low intensity, low power, low level, low energy, cold, soft, non- therrial, biostimulation, biomodulation, or photobiostimulation laser, etc.

Trying to use a common language, we agree with the opinion of the professor Atsurni to use only "Low Energy Laser (L.E.L.)". We think that it is an accurate terminology and it underlines the main character of laser : an energetical source.

\section{Kinds of low energy laser (L.E.L.) used in medicine.}

They are: HeCd (444nm), Argon (488-515nm), CO2 (10600nm), etc.

The most used lasers are:

a) Helium-Neon (HeNe)-wavelength $632 \mathrm{~nm}$, with continuous emission in visible spectrum in red

b) Galium-Aluminum-Arsenide (GaAlAs)-wavelength $820-904 \mathrm{~nm}$, with pulsed emission $(100-200 \mathrm{~ns})$, in infrared.

For the therapeutical purposes wavelength and power (output power) seem to be important.

\section{Medical field of L.E.L. application.}

The most important are: basic research, diagnosis (see table 1), rehabilitation (laser sensory prothesis, laser for blind, holographic spectacies) and therapeutics (see table 2). 
Table 1

\begin{tabular}{|l||l|}
\hline Basic research & Diagnosis \\
\hline photodynamic effects & laser illumination \\
\hline cultured cell & spectral analysis \\
\hline action on cancer cell & measurements(e.g.retinal resolution) \\
\hline reactivation of enzymes & laser Doppler method \\
\hline bactericidal effect & laser microscope \\
\hline action on infracellular structures & holography (retinal imaging, etc) \\
\hline others & others \\
\hline
\end{tabular}

We will direct attention on the therapeutics field and especially to the first five indications. We aiso refer to the basic researches.

Table 2.

\begin{tabular}{|l|}
\hline \multicolumn{1}{|c|}{ Therapeutics } \\
\hline \hline antiphlogistic reaction and rheumatic diseases \\
\hline pain relief \\
\hline wound and bone-fractured healing \\
\hline hormonal and neural stimulation \\
\hline laserpuncture \\
\hline photodynamic therapy \\
\hline dentistry \\
\hline others \\
\hline
\end{tabular}

Medical therapeutics can be divided in two important groups, in function on the main effect:

1.Photodynamic effects: photodynamic therapy, activation of the enzymes, hormones, vitamins and also bactericidal effects.

2.Electromagnetic effects: the others already mentioned.

It is important to underline that we can use laser therapy clinically in many diseases, but having always in our mind the fact that this therapeutical system is not unfailing (some kind of magic), and is not to be used for all kinds of diseases. For example, we can treat many rheumatic diseases, but it is less efficient in inflammatory ones; different kind of pain respond well, but not the pain of cancer origin, etc.

\section{The characters of the laser light and their importance.}

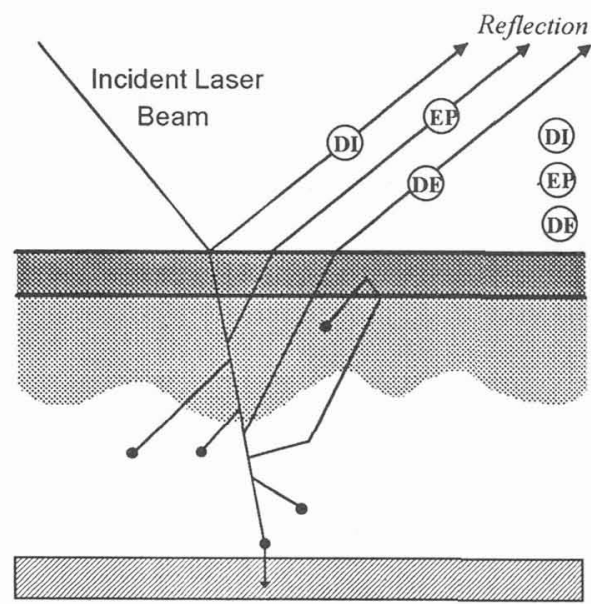

Picture 1 Reflection of the laser beam on the skin
a)Coherency is always present as a character of laser, but differs in different kinds of lasers. In deeper penetration through living tissues, coherency decreases. It seems that coherent light induces local microcirculation. There are many papers showing that this character is not absolutely necessary to obtain good effects, but we don't agree.

b) Polarization in space is only one component of laser and it has stimulatory effect, e.g. on the activity of the lymphocytes, but even without it the other character of laser light manifest biostimulatory effects. Although it is better, if possible, to perform direct laser beam instead of optic fibers.

c) Monochromatic

character seems to be extremely important, and as the light is less monochromatic, as the effects are poorer. We don't think that this light character is more important than the particular photon energy (e.g. its wavelength). 
d) Direction of the laser is as an parallel beam which makes the dose estimation easy and safe.

e)Penetration is different depending on the wavelength of the laser type.

For example, HeNe laser is highly monochromatic, highly polarized and with excellent coherence, and is good for general use, but has no deep penetration in tissues.

The Argon laser has a shorter penetration, but CO2 laser has the shortest one, both of them being used in superficial desinfection and wound or various ulcer healing.

The infrared laser has the best penetration in tissues (around $4 \mathrm{~cm}$ ), but with less absorption in the superficial tissues.

The angle of application of the laser beam on the skin is important (see picture 1).

Reflection is a direct one and also originates from epiderm,derm and subcutaneous tissues.

For an orthogonal application, the reflection (remittance) at the stratum corneum is of $4-7 \%$, $93-96 \%$ of the laser radiation being absorbed in the underlying tisstes. The limit angle of the laser application must be over 41 grade, because under this value almost total reflection will be produced.

\section{Hypothesis of the L.E.L, action.}

Owing to the big number of hypothesis and lack of space, we cannot list them all. We tried to present an unitary and ordered picture. There are some very important papers referring to this subject, and we have to mention their authors (in alphabetical order): Atsumi, Bolognani, Costato, Karu, Kamikawa, Mester. All of them and many others have contributed to clarify many aspects of the L.E.L. mechanisms of action.

L.E.L. biostimulation may act, dose and wavelength depending, stimulating or inhibiting, at the following levels: a)Infracellular b)Cellular c)Systemic (Organism).

\section{A.Infracellular level.}

At this level may act the so called "informational resonance phenomenon" between the coherent and monochromatic electromagnetic source frequency and the cellular dipole frequency. The interference between the two sources, the armonic or disarmonic generation and the electronic excitation or deexcitation status may be considered as a resonance mechanism.

The L.E.L. dissipation at the infracellular level may alter the molecules only in a reversible way. We suggest that the molecular reversible alteration is the most important component of the photophysical changes in the biological target.

In this case the energy transfer can be done through movements of further electromagnetic waves, transfer of particles, charge transfer and conduction.

We also suggest that the "biostimulation effect" of the L.E.L. operate mainly at the infracellular level.

\section{B.Cellular level.}

At this level, the L.E.L. activates the main target : cell wall, cell nucleus and the mitochondria (the major mediator of laser effects). We proposed a cascade of events (in or after laser stimulation):

-optimization of the Na-K cell membrane pump

-stabilization of the lysosome membrane

-activation of the mitochondrial functions

-activation of the inactivated enzymes (Krebs cycle, oxidation chain, etc).

-higher rate of ATP production and higher rate of bioenergetic potential

-activation of the DNA-RNA-protein system

-increased protein synthesis

-higher rate of mitosis

-stimulation of reproduction, reparative regeneration, activation of the phagocites and increasing resistance to pathogenic factors.

\section{C.System (Organic) level.}

The main effects of L.E.L. application "in vivo"may lead to:

-keeping the omeostasis of the energetic equillibrum

-activation of the neuroendocrin system

-stimulation of the immune system (umoral specific immunity and nonspecific defense system)

-activation of the hemopoiesis mechanism 
-activation of the antiinflammatory mechanism

-improved microcirculation as well as vascularization of blood vessels, vassodilatation or vascular anastomosis

-improved tissue granulation and epitelization

-neural stimulation

-citotoxic (antibacterial) effect

-bone-fracture healing

-dental effect

-stimulation of general adaptative reactions of the organism (anti-stress reaction).

\section{Practical methodology.}

Some authors recommend to calculate the irradiation dose using this formula:

$$
\mathbf{E}=\mathbf{P} * \mathbf{T}
$$

where:

\section{E-energy (Joule) P-power (Watts) T-time (seconds)}

be:

For example, in case of an HeNe laser with a power of $10 \mathrm{~mW}$, in 2 minutes, the energy will

This is:

$$
E=0.01 W * 120 \mathrm{~s}=1.2 \mathrm{~J}
$$

In case of $\mathbb{R}$ laser with a pulsed emission it is more important to know the output power.

$E=$ Peak Power * No of impulses * Time of one impulse

Another parameter is the density of energy: Energy (Joule) / Surface $\left(\mathrm{cm}^{2}\right)$

This is an useful kind of common language, but sometime we have to mention both the power and the time of exposure, because in clinical practice is not the same thing if we use a high power for a short time or a low power for a long time. Also for IR laser, the point is rather different, as we mentioned above.

We must do some remarks about basic or clinical use:

-the angle for irradiation must be as close of the rectangular as it could be

-cleaning the skin with alcohol it is not so important as some authors mentioned

-the irradiation must not be used on some dermatological lesions (like cancer lesions) beam

-the eyes of the patient and our eyes must be protected from the direct action of the laser

-the irradiation either by HeNe or by GaAlAs laser can be done stimulating some points (acupunctural points, loco-dolenti or other points) for a certain period (seconds, minutes) or in an "active" way, scanning the surface we need to irradiate or moving the laser beam (especially for IR laser) along some circuits, which vary depending on specific disease. The number of stimulated points could be 1-20 (even more), the irradiation time per point could be 30 seconds-10 minutes, with sessions twice a day, every day, every two days (a better solution), every three days, once a week, etc.

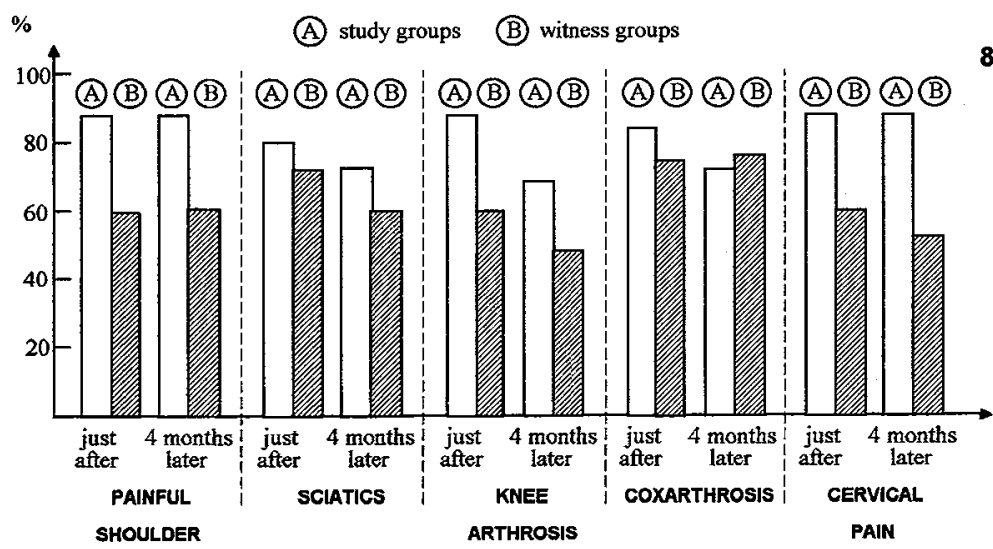

Picture 2 - Percentage evaluation of the treatment efficiency for different diseases, just after the treatement and 4 months later

8.Our clinical
experience.

First of all, we must say that we always compared the clinical group of patients with a witness group treated by placebo or by classical therapeutical

means, in a simple or double blind studies. We worked with a $\mathrm{HeNe}$ laser with a power of 2 $\mathrm{mW}$ and a GaAlAs laser with an output power of 1-2 $\mathrm{mW}$ together or isolated.

We used the two kinds of lasers in the treatment of 340 patients with rheumatic diseases ( or 
pains) like : gonarthrosis, shoulder periarthritis, lumbago and sciatics, coxarthrosis and cervical spondilosis (cervical neuralgia).

We obtained good results in over $75 \%$, excepting coxathrosis, where the efficiency was smaller (63\%) (see picture 2).

In another study we describe the results of the treatment with low energy laser (GaAs and/or $\mathrm{HeNe}$ ) single, or in combination with classical therapy on 148 female patients with pelvic inflammatory diseases, and we obtained good results in over $82 \%$, especially using both lasers simultaneously.

We also had good results in the treatment of 64 patients with posttraumatic swellings, varicose, crural ulcer and less good in allergic vasculitis (see picture 3).

Now we are studying the efficiency of L.E.L. therapy in the recovery of some patients with traumatic lesions of median nerve. The first results are encouraging.

We must say that for a clinician a big problem is how to objectivize, in a satisfactory manner, the clinical results.

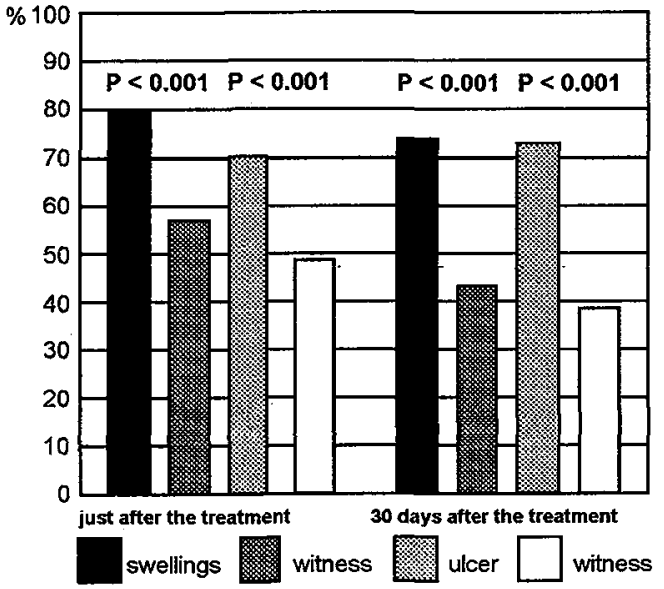

Picture 3 - The efficiency of the treatment (\%)

\section{Proposals of scientific standards for comparative clinical studies.}

Low laser therapy has an increasing popularity as a new therapy for many disorders, in spite of a shortage of objective controlled studies. That means the scientific standardization is imperiously necessary. This kind of criteria must assure the accuracy and the reproducibility of the scientific methods and studies. Even if what we will present could seem well known or useless, we think that those who have studied this scientific domain felt this necessity.

We also want to underline that it is necessary to be cautious in extrapolating results from "vitro" to "in vivo" and from animal to human.

\section{A.Criteria for L.E.L. sources standardization.}

-spectral region of laser radiation

-wavelength (nm)

-output power (mJ)

-modality of laser irradiation: continuos exposure laser, discontinuous exposure laser, pulsed laser, repetitively pulsed laser, variable exposure laser

-single wavelength and multiple one

-diameter of the laser spot on stimulated point

\section{B.Criteria for L.E.L. therapy regimens.}

-frequency of the laser administration: daily, twice a day, every two days, once a week, etc -therapeutical modality of laser irradiation: only per points, laser acupuncture, active, scanning, combination

-total output energy per irradiation areas (points)

-total exposure time (per point, active, scanning)

-total number of points per session-topographic map of irradiation areas

-total number of sessions for specific diseases.

\section{C.Criteria for patient groups and control groups..}

-the studies must be double blind (at least simple blind)

-the treatment groups will contain:

- a laser therapy group

-a placebo laser therapy group

-a drug (or other physical therapy) group 
-possibly a self control group ( for individual variation study, each patient must be his own witness)

-the patients group must be homogenous in age, sex, clinical and paraclinical parameters, according to widely accepted criteria.

\section{D.Objective and subjective improvements analyze of the laser treatment. .}

One of the most difficult problems of the laser therapy is to find an objective method to measure the efficiency of the treatment so, we must :

-to establish the minimum objective criteria for each disorder in study

-to evaluate the major symptoms (e.g. pain) on a visual analogue scale and the main signs

-to compare the differences between the groups mentioned above immediately after treatment and also after months or even years after the treatment, because we noticed that sometime the efficiency of the laser therapy diminished in time.

-to apply for all the comparisons in the studies, the same type of statistical method.

Table 3.

\begin{tabular}{|c|c|}
\hline Contraindications & Side-effects \\
\hline laser applications at the retinal level & \multirow{2}{*}{$\begin{array}{l}\text { increasing of the pain after the } 1 \text { st or } 2 \text { nd laser } \\
\text { session, usually decreasing after } 1 \text { or } 2 \text { days }\end{array}$} \\
\hline hyperthyroidism & \\
\hline cancer (especially of the skin) & increasing of the menstrual flow \\
\hline mastopathys & nausea and other dyspeptic symptoms \\
\hline acute bacterial infections & \\
\hline pregnancy (lack of studies) & \\
\hline cardiac aritmias,carriers of pace-makers & \\
\hline
\end{tabular}

Finally, we want to underline that L.E.L. represents a new therapeutical method, with a few contraindications or side- effects(see table 3), efficient and cheap, which is winning more and more new supporters.

The studies of the acting mechanism of laser therapy is following not only to establish a phisiopathological basis in order to issue the most accurate and correct treatment, but also to discover the modalities of energetical or informational transfer between cells (cell-to-cell transmission) or inside the organism.

\section{ACKNOWLEDGMENTS.}

The authors thank to Franco-Romanian Bank for supporting travel expenses.

\section{References (a selection).}

1.C.Antipa,D.Dona,A.Podoleanu -"Use of pulsed laser therapy (GaAs) in combined treatment of post traumatic swellings and some dermatological disorders", Biomedical Optic's, Europe, Budapest,1-5 sept 1993.

2.C.Antipa,D.Dona,A.Podoleanu,N.Crisan,C.Constantinescu -"Laser's biostimulation (heNe and GaAs) effects as compared to the conventional therapy in several pelvic inflammatory diseases", Biomedical Optic's 93, Los Angeles, 20-25 ian 1993.

3.A.Podoleanu,C.Antipa -"GaAs Laser Rheumatismal Biostimulotherapy", in J. de Physique,IV, vol 1, C7-231,1991.

4."Laser Application in medicine and surgery" (Galletti,Bolognani,Ussia edit) 1992:

-K.Atsumi -"The state of the art of low power laser in medicine",p.5.

-F.Bersani -"The physics of electromagnetic waves",p.197.

-L.Bolognani,N.Volpi -"Low power laser-enzyme interaction",p.213

-L.Bolognani,N.Volpi -"Laser interaction with biosystems at cellular and molecular level",p.235

-G.Castronuovo,G.Fava,S.Giavelli -"The skin role during a low level laser therapy",p.19

-M.Costato -"Laser light and the biological response: cooperative phenomena vs order and disorder",p.191

-Z.G.Horvath,Z.Donko -"possible ab-initio explanation of laser <<biostimulation>> effects",p.11

-T.Karu -"Fundamentals of interaction of visible and near infrared low-intensity laser radiation with cells",p.203

-A.R.Mester -"Modalities of low power laser applications",p.33

-S.Passare/la -"LPL radiation of mitochondria and mitochondrial enzymes",p.223.

- C.Shiroto - "Clinical results of pain relief with low level laser therapy",p.25 